ACCEPTED MANUSCRIPT

\title{
Electrospinning of highly concentrated albumin patches by using auxiliary polymers for laser-assisted vascular anastomosis
}

To cite this article before publication: Annemarie Schönfeld et al 2018 Biomed. Mater. in press https://doi.org/10.1088/1748-605X/aac332

\section{Manuscript version: Accepted Manuscript}

Accepted Manuscript is "the version of the article accepted for publication including all changes made as a result of the peer review process, and which may also include the addition to the article by IOP Publishing of a header, an article ID, a cover sheet and/or an 'Accepted Manuscript' watermark, but excluding any other editing, typesetting or other changes made by IOP Publishing and/or its licensors"

This Accepted Manuscript is @ 2018 IOP Publishing Ltd.

During the embargo period (the 12 month period from the publication of the Version of Record of this article), the Accepted Manuscript is fully protected by copyright and cannot be reused or reposted elsewhere.

As the Version of Record of this article is going to be / has been published on a subscription basis, this Accepted Manuscript is available for reuse under a CC BY-NC-ND 3.0 licence after the 12 month embargo period.

After the embargo period, everyone is permitted to use copy and redistribute this article for non-commercial purposes only, provided that they adhere to all the terms of the licence https://creativecommons.org/licences/by-nc-nd/3.0

Although reasonable endeavours have been taken to obtain all necessary permissions from third parties to include their copyrighted content within this article, their full citation and copyright line may not be present in this Accepted Manuscript version. Before using any content from this article, please refer to the Version of Record on IOPscience once published for full citation and copyright details, as permissions will likely be required. All third party content is fully copyright protected, unless specifically stated otherwise in the figure caption in the Version of Record.

View the article online for updates and enhancements. 


\title{
Electrospinning of highly concentrated albumin patches by using auxiliary polymers for laser-assisted vascular anastomosis
}

\author{
Annemarie Schönfeld ${ }^{1,2}$, Mihai Constantinescu ${ }^{3}$, Kirsten Peters ${ }^{2}$, Martin Frenz ${ }^{1}$, $^{*}$ \\ ${ }^{1}$ University of Bern, Institute of Applied Physics, Department of Biomedical Photonics, Sidlerstrasse 5, Bern, Switzerland, 3012 \\ ${ }^{2}$ University Medicine Rostock, Department of Cell Biology, Schillingallee 69, Rostock, Germany, 18057 \\ ${ }^{3}$ University Hospital Bern, Department of Plastic and Reconstructive Surgery, Bern, Switzerland, 3010
}

\begin{abstract}
.
Background and Objective: Electrospun meshes have been extensively investigated for tissue engineering and drug delivery. The application of this technology is of interest for laser-assisted vascular anastomosis (LAVA) due to the possibility to bind and stabilize macromolecules in fibers.

Materials and Methods: We prepared bovine serum albumin (BSA) blend microfibers from the auxiliary proteins polyethylene oxide (PEO), polycaprolactone (PCL), polyvinyl alcohol (PVA) and gelatin. The thickness and weight of the resulting patches were measured and the morphological characteristics were observed by scanning electron microscopy. Thereafter, layered patches were prepared by spinning the BSA/polymer layer on top of a light-absorbing layer made of indocyanine green and PCL. The effect of the material composition of the electrospun patches on the behavior during LAVA, the bonding strength and the resulting thermal damage were investigated.

Results: The bonding strength of the tissue fusion increased with higher BSA amounts in the patch. By using PEO, a ratio of 85/15 (w/w) of BSA/PEO was stable during electrospinning, leading to a shear strength that was similar to patches that were soaked in liquid BSA $\left(20.7 \pm 4.1 \mathrm{mN} / \mathrm{mm}^{2}\right.$ and $20.3 \pm 4.1 \mathrm{mN} / \mathrm{mm}^{2}$, respectively). The handling during LAVA was however drastically improved by using a layered patch made from BSA/PEO. Thermal damage was similar compared to previous solder materials. Conclusion: This study investigated the maximum amount of BSA possible in electrospun polymer fibers made from PEO, PCL, PVA and gelatin. Both, the process of electrospinning and the performance during ex vivo LAVA, makes the BSA/PEO blend a promising material for LAVA.
\end{abstract}

Keywords: indocyanine green, in vitro studies, shear strength, tissue fusion, tissue soldering.

*Corresponding author, frenz@iap.unibe.ch

\section{Introduction}

Laser-assisted vascular anastomosis (LAVA) has potential to enable secure, rapid and easy fusion of blood vessels and might reduce suture and needle trauma, foreign body reactions and bleeding $[1,2,3]$. LAVA is based on the inauguration of a thermal effect driven by the absorption of laser light, inducing denaturation and coagulation of proteins. Solder that commonly consists of the light absorbing chromophore indocyanine green (ICG) and protein bovine serum albumin (BSA), is applied onto the soldering site by either dripping $[3,4,5]$ or by soaking a patch in BSA/ICG solution. For the latter, polymers such as chitosan [6], PCL [7, 8] and poly(DL-lactic-co-glycolic acid) [9], have been investigated. However, the usage of a liquid solder solution has disadvantages: (1) Dripping of the liquid solution before and during soldering lead to an uncontrollable amount of both, the chromophore and the protein. (2) The difficult localized deposition of liquid solder and the flow-off of the solder, results in staining surrounding tissue and hence heating and excessive thermal damàge. (3) Applying solder solution, containing highly concentrated BSA, is a tedious process, due to the stickiness of the solution. (4) The short shelf-life of highly concentrated BSA solution necessitates its fresh preparation prior to surgery to avoid protein degradation and aggregation [10]. A patch, that is soaked in liquid BSA/ICG solution, followed by application to blood vessels, possesses the same disadvantages with regard to liquid highly concentrated BSA solutions, but significantly increases the bonding strength. The increased bonding strength is a result of the larger surface area that is involved in the bonding due to the fusion of the solder patch along the sides of the tissue and not only at the edges of the tissue to be fused, as for liquid solder $[6,7,8]$. Our aim is thus to develop and characterize a pliable and tear-proof patch that already encapsulates both ICG and BSA, that is biocompatible and biodegradable, and is ready-to-use without additional modifications in the surgery room. 
Electrospinning is a well-studied technique to produce fibers with diameters in the nano- and micrometer range [11]. Electrospun materials can be prepared from a large variety of synthetic or natural polymers, enabling a wide field of applications such as in filtering [12], sensors [13] or textiles [14]. Powerful progress has been achieved in using electrospinning for tissue engineering $[15,16,17,18]$, wound dressing $[19,20$, $21]$, and the encapsulation of drugs [22, 23, 24, 25, 26] and cells [27, 28].

The application of an electrospun scaffold made of ICG-loaded polycaprolactone (PCL) fibers that was soaked in liquid BSA has been shown to improve tissue fusion [29]. To obtain strong tissue fusion during LAVA, the amount of BSA must be high and the BSA layer needs to have direct contact with the underlying tissue ensuring the denaturation and subsequent entanglement of the protein chains $[4,30]$. We present a double-layer patch, with the BSA-loaded layer touching the tissue and with the PCL/ICG-absorber layer above. As stated in the literature, the protein BSA does not form fibers during electrospinning, due to its globular structure. To overcome this limitation, blend [31, 32, 33, 34], coaxial [35, 36] and emulsion $[37,38,39]$ electrospinning have been shown to incorporate biomolecules into fibers.

The present study addresses the development of scaffolds with a high BSA-load via blend electrospinning together with an auxiliary polymer. BSA-loaded layers from four different auxiliary polymers and with different weight ratios were prepared and investigated with regard to weight, thickness and fiber morphology. The following biocompatible polymers were tested: polyethylene oxide (PEO), polyvinyl alcohol (PVA), gelatin and PCL. PEO is a biocompatible polymer, water-soluble and known for its good electrospinnability [31, 40, 41]. Protein-loaded PVA nanofibers have been investigated for application in biosensors [33], tissue engineering [42], and protein delivery, systems [43,44]. Gelatin is the irreversibly hydrolyzed form of collagen, which is a naturally abundant polymer. Gelatin was frequently used in tissue engineering to serve as a matrix material to provide support for cells [45, 46, 47, 48], and could therefore be a potential candidate for LAVA. PCL is shown to be suitable as a matrix polymer for the ICG-loaded layer [29] and was therefore investigated as an auxiliary polymer for BSA-electrospinning. The solution and electrospinning parameters were optimized in order to ensure an electrospinning process without dripping of the solution or clogging of the needle tip and to ensure the production of smooth membranes. The applicability of the layered solder patches to LAVA was tested in vitro using rabbit aortas. The process was controlled with an infrared camera and the impact of the solder patches was determined by measuring the shear strength of the anastomoses and thermal damage assessment using histologies.

\section{Materials and Methods}

\subsection{Materials}

Polycaprolactone (PCL, $\mathrm{M}_{n}=70000$ to 90000), polyethylene oxide (PEO, average $\mathrm{M}_{n}=400000$ ), polyvinyl alcohol (PVA, $\mathrm{M}_{n}=205000$ ), gelatin (from porcine connective tissue, bloom $175 \mathrm{G}$ ), acetic acid (p.a.), formic acid (p.a.), and lyophilized bovine serum albumin (BSA) were obtained from Sigma Aldrich (Saint Louis, MO). Methanol and chloroform were used in analytical grade from Merck KGaA (Darmstadt, Germany). Indocyanine green (ICG, IR-125) was obtained from Acros Organics (Geel, Belgium). All materials were used as received.

\subsection{Preparation of patches}

\subsubsection{Electrospinning of BSA-loaded patches}

The electrospinning solutions were prepared by simultaneously adding specific amounts of the polymer and BSA to the solvent mixture, see table 1 . The solutions were stirred overnight at $37^{\circ} \mathrm{C}$. Polymer and BSA concentrations are expressed in $\%(\mathrm{w} / \mathrm{w})$ relative to the solution.

For electrospinning, the prepared solution was placed in a $5 \mathrm{ml}$ syringe that was connected with Teflon tubings to a truncated needle $(21 \mathrm{G}, 0.812 \mathrm{~mm}$ I.D.). A flow rate of $10-30 \mu \mathrm{l} / \mathrm{min}$, a collector distance of 10 $30 \mathrm{~cm}$ and a positive potential of $5-25 \mathrm{kV}$ were employed, depending on the stability of the electrospinning 
process. The fibers were collected on a rotating aluminum plate to aid even distribution of the deposited fibers. The humidity and the temperature were fixed at $30 \pm 5 \% \mathrm{RH}$ and $22 \pm 1{ }^{\circ} \mathrm{C}$.

\subsubsection{Electrospinning of ICG-loaded layer}

A $9 \%(w / w)$ PCL solution with ICG at a ratio of 1:10 (w/w) ICG:PCL was prepared in chloroform/methanol $(75 / 25(\mathrm{v} / \mathrm{v}))$. The blend was electrospun for $75 \mathrm{~min}$ using a positive potential of $15 \mathrm{kV}, 15 \mathrm{~cm}$ collector distance and a flow rate of $30 \mu \mathrm{l} / \mathrm{min}$.

\subsection{Analysis of the patches}

\subsubsection{Scanning electron microscopy (SEM)}

To examine the fiber morphology, the patches were cut into small pieces, sputter coated with a $10 \mathrm{~nm}$ layer of gold and imaged using scanning electron microscopy. The fiber diameters were analyzed using FIJI (ImageJ 1.51a) [49]. The average fiber diameter and standard deviation were determined by preparing 3 patches and measuring the cross section of 60 fibers per image $(n=180)$.

\subsubsection{Weight and thickness}

After determining the solution and process parameters that led to stable electrospinning, the BSA/polymer blend was electrospun directly on an ICG-loaded layer to produce a solder patch. From each blend two solder scaffolds with a diameter of $10 \mathrm{~cm}$ were electrospun, from which 5 solder patches were cut and used for the thickness, weight, and tensile strength measurements $(n=10)$. The size of the solder patches was $10 \pm 1 \mathrm{~mm}$ times $20 \pm 1 \mathrm{~mm}$. The weight of the ICG-loaded layer was determined to be $9.1 \pm 2.3 \mathrm{mg}$ ( $\mathrm{n}=50$ ICG-layers) and subtracted from the weight of the layered solder patch. For the determination of the thickness of the layers, a layer was inserted between two microscope slides, followed by measuring the displacement with a height gauge (Trimos V600+, Trimos SA, Renens, Switzerland). The tensile strength measurement of the layers is described in chapter 2.3.4.

\subsubsection{In vitro laser-assisted vascular anastomosis}

The applicability of the different solder patches was tested using the LAVA technique shown in figure $1 \mathrm{~A}$. Rabbit aortic arteries from a local slaughterhouse were liberated from surrounding fatty tissue, cut transversal and the two stumps were placed onto a water-filled balloon catheter (Armada 35 PTA catheter, Abbott, Illinos). A diffusor laser fiber with a $15 \mathrm{~mm}$ active zone (Laser und Medizintechnologie Berlin, Germany) was inserted into the catheter. An $810 \mathrm{~nm}$ diode laser (Lina 30d, Intros, Heilbad Heiligenstadt, Germany) was coupled into the laser fiber. The solder patches were then centrally placed on the arteries, in such a way that the patch covered $5 \mathrm{~mm}$ of both vessel stumps and with the BSA-loaded layer in direct contact with the vessel wall. The patch was rolled over the arteries with an overlap of $2 \mathrm{~mm}$. Subsequently, the vessel was irradiated using an average output power of $3 \mathrm{~W}$, in the continuous wave $(\mathrm{cw})$ regime. The vessel diameter was measured to be $3.9 \pm 0.3 \mathrm{~mm}(\mathrm{n}=30)$, thus the irradiated surface was $1.8 \pm 0.1 \mathrm{~cm}^{2}$, resulting in an irradiance of $1.6 \pm 0.2 \mathrm{~W} / \mathrm{cm}^{2}$. During soldering, the temperature on the surface of the patch was measured with a thermal camera (A655, FLIR systems, Oregon, USA) and manually controlled by switching the laser on and off. After reaching a value of $75^{\circ} \mathrm{C}$ we irradiated for further $30 \mathrm{~s}$ at a temperature range between $75^{\circ} \mathrm{C}$ and $85^{\circ} \mathrm{C}$.





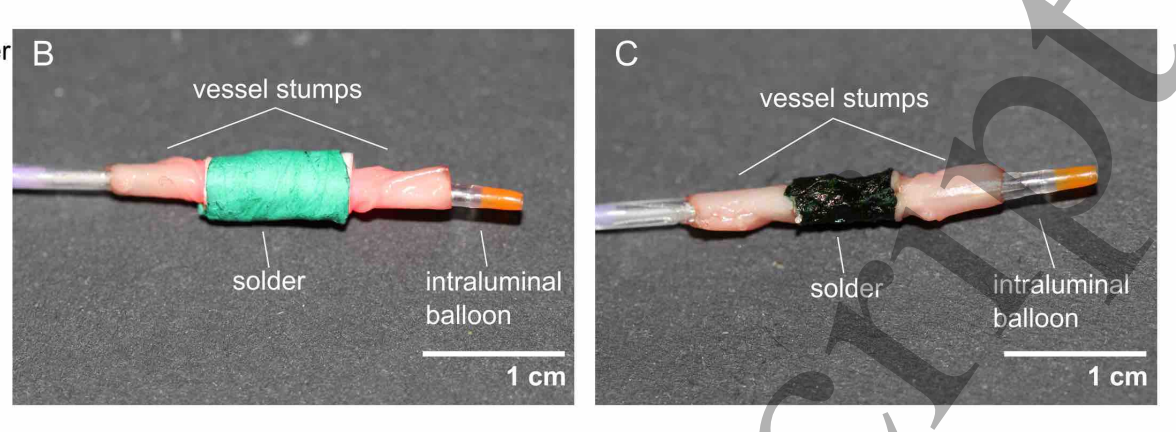

Fig 1 A: Schematic of the LAVA setup (adapted from Ott et al. [3]). B: Experimental setup showing the aligned vessel stumps in vitro with the balloon catheter and the patch that was wrapped around the vessel before irradiation. C: LAVA after irradiation. A color change towards dark-green and radial shrinkage was observed.

\subsubsection{Measuring shear strength}

The quality of the fusion was assessed by measuring shear strength. Therefore, a test stand with a fixed force gauge (BFG50, Mecmesin Limited, West Sussex, United Kingdom) was employed. The vessels were fixed with two surgical clamps $2 \mathrm{~mm}$ from the ends of the patches. The clamps were connected to a moving table, which was pulled with an electrically driven motor at a constant velocity of $30 \mathrm{~mm} / \mathrm{min}$ and the maximum load at the failure of LAVA was recorded. The applied test force is perpendicular to the normal of the soldered surface. Since only one of the two vessel stumps eventually slips off, the shear strength was calculated as the maximum load divided by half the patch area. The shear strength was reported in $\mathrm{mN} / \mathrm{mm}^{2}$. Similarly, the tensile strength of the electrospun layers before soldering was measured, where the maximum load was divided by the thickness times the width of the layer ( $n=5$ layers).

\subsubsection{Histological Analysis}

The thermal damage after LAVA applying the layered patch with BSA/PEO (85/15 (w/w)) was histologically assessed. Therefore, rabbit aortas were obtained from the slaughterhouse, transported on ice, and processed within 4 hours. Immediately after soldering, the tissue samples were fixed in $4 \%$ formaldehyde (G256, Dr. Grogg Chemie AG, Stettlen, Switzerland) and routinely processed in methylmethacrylate (MMA). Therefore, the samples were rinsed in water, dehydrated in ascending ethanol fractions, infiltrated and embedded in MMA. The embedded blocks were serially cut into $500 \mu \mathrm{m}$ thick sections using a diamond saw. Subsequently, the specimens were mounted onto acrylic glass slides, and the sections were ground to a final thickness of $80 \mu \mathrm{m}$ and stained superficially with toluidine blue combined with basic fuchsin. For higher resolution, the specimen were subdivided into smaller samples and embedded in epon resin (Fluka, Buchs, Switzerland). Sections were cut and stained with toluidine blue and basic fuchsin, and studied using a Zeiss Axioplan microscope (Carl Zeiss GmbH, Jena, Germany).

\subsubsection{Statistical Analysis}

Values are reported as mean \pm standard deviation. The non-parametric Wilcoxon rank test was performed to compare the shear strength resulting from soldering with the different polymer patches. The tests were performed with R 3.4.2 [50].

\section{Results}

\subsection{Optimization of solution and electrospinning parameters}

Four polymers were electrospun and applied to LAVA. Table 1 lists the BSA/polymer ratios and the process parameters used. 
Table 1 Solution and process parameters used for electrospinning of the BSA/polymer blends and the appearance of the process and the patch.

\begin{tabular}{lllllllll} 
PEO & 7.4 & 1.3 & water & 15 & 15 & 15 & stable & homogeneous, no flaws \\
& 4.4 & 4.4 & 15 & 15 & 15 & stable & homogeneous, no flaws \\
\hline PVA & 7 & 7 & water & 20 & 15 & 20 & rarely dripping & homogeneous, few droplets \\
\hline Gelatin & 10 & 20 & formic acid & 15 & 15 & 10 & stable & homogeneous, no flaws \\
\hline PCL & 6 & 10 & formic/acetic & 22 & 15 & 15 & dripping & hormogeneous, no flaws \\
& 20 & 3.5 & acid 70/30 v/v & 22 & 15 & 15 & dripping & homogeneous, few droplets \\
\hline
\end{tabular}

\subsubsection{Polyethylene oxide (PEO)}

As a starting point, a blend of $7.4 \%(\mathrm{w} / \mathrm{w}) \mathrm{BSA}$ and $1.3 \%(\mathrm{w} / \mathrm{w})$ PEO was used as published by Kowalczyk et al. [31]. Stable electrospinning of this blend was achieved by applying a gap distance of $15 \mathrm{~cm}$, a feed rate of $15 \mu \mathrm{l} / \mathrm{min}$ and an applied voltage of $15 \mathrm{kV}$. The solution did neither drip nor clog at the needle tip, and spinning for hours was possible without any adjustment. The appearance of the resulting patch was homogeneous, without any clumping. With the BSA/PEO ratio being 85/15 (w/w), the amount of BSA in the final layer was $10.1 \pm 1.6 \mathrm{mg}$. Experiments involving different BSA amounts, revealed that the shear strength of the tissue fusion increased with increasing BSA amount. A BSA amount of $10.1 \pm 1.6 \mathrm{mg}$ in the patch was the highest obtained during BSA-blend electrospinning. Figure 2 shows the morphology of the electrospun fibers made from the different polymer/albumin blends. The electrospun nanofibers made from BSA/PEO $(85 / 15(\mathrm{w} / \mathrm{w}))$ resembled a string of beads. Electrospinning with a lower BSA and higher PEO concentration $(4.4 \%(\mathrm{w} / \mathrm{w}) / 4.4 \%(\mathrm{w} / \mathrm{w}))$ resulted in a solution of higher viscosity and in fibers. Scaffolds made from PVA and gelatin show random fibers with a mean diameter of $412 \pm 75$ and $494 \pm 48 \mathrm{~nm}$, respectively. Fibers made from PCL were in the same range, being $396 \pm 147 \mathrm{~nm}$, if spun from a solution of BSA/PCL 6/10\% (w/w) and significantly thinner if spun from a solution of BSA/PCL 20/3.5\% (w/w), being $153 \pm 63 \mathrm{~nm}$.

\subsubsection{Polyvinyl alcohol (PVA)}

By using different BSA/PVA ratios with water as a solvent, BSA/PVA fibers were only obtained at PVA concentrations of $7 \%(\mathrm{w} / \mathrm{w})$. At lower concentrations, a consistent jet could not be produced, and the solutions were constantly dripping. Using a blend of $7 \%(\mathrm{w} / \mathrm{w})$ BSA and $7 \%(\mathrm{w} / \mathrm{w})$ PVA, a homogeneous layer, with a few droplets, was obtained. However, after varying the gap distance, voltage and feed rate, rarely dripping of the solution from the needle tip could not be avoided. This led to a significant loss of material. By further increasing the BSA ratio, BSA and PVA did not dissolve to a homogeneous blend.

\subsubsection{Gelatin}

We tested various BSA/gelatin ratios in order to receive a homogeneous layer with a high amount of BSA. BSA/gelatin blends in formic acid produced homogeneous scaffolds at 20 and $10 \%$ (w/w), respectively, and the jet formed during electrospinning was uniform and consistent. Application of higher BSA amounts led to clogging of the needle tip. 


\subsubsection{Polycaprolactone (PCL)}

For the ICG-loaded layer, PCL was diluted in chloroform/methanol, which led to stable electrospinning. For blend electrospinning with BSA, another solvent than chloroform was required, as BSA denatured in this solvent. Roozbahani et al. [51] demonstrated electrospinning of PCL and BSA in a formic acid and acetic acid solvent system. In our study, a homogeneous layer was achieved by using a blend made of $10 \%(\mathrm{w} / \mathrm{w})$ PCL and $6 \%(\mathrm{w} / \mathrm{w})$ BSA, with some droplets falling from the needle tip. By decreasing the polymer concentration to $3.5 \%(\mathrm{w} / \mathrm{w})$, the BSA concentration could be increased to $20 \%(\mathrm{w} / \mathrm{w})$, with the same electrospinning behavior as the blend above.

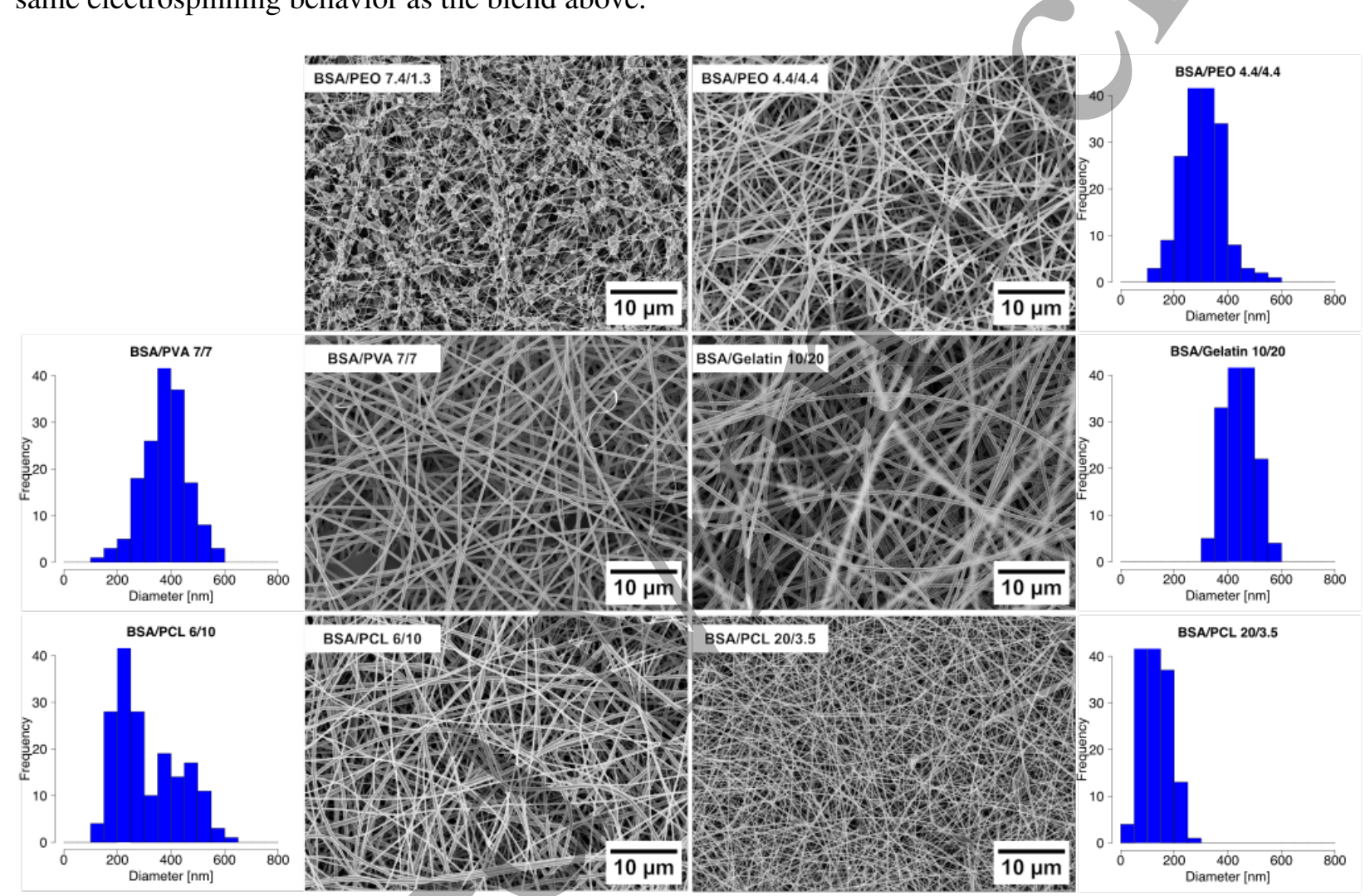

Fig 2 Fiber morphology of the electrospun polymer and albumin blends. BSA/PEO fibers (7.4/1.3\% (w/w)) display a beadson-a-string morphology. All other polymer/BSA solutions produced random, straight fibers, with the following fiber diameters: BSA/PEO (4.4/4.4\% (w/w)) 315 $\pm 62 \mathrm{~nm}$; BSA/PVA (7/7\% (w/w)) 412 $\pm 75 \mathrm{~nm}$; BSA/gelatin $(10 / 20 \%(\mathrm{w} / \mathrm{w})) 494 \pm 48 \mathrm{~nm}$; BSA/PCL (6/10\% (w/w)) 396 $\pm 147 \mathrm{~nm}$; BSA/PCL (20/3.5\% (w/w)) $153 \pm 63 \mathrm{~nm}$. The distribution of the fiber diameter is shown left and right from the corresponding micrographs. The frequency of diameters from 180 fibers are displayed per histogram.

\subsection{Preparation of patches: electrospinning duration, thickness, weight, and tensile strength}

Layered BSA- and ICG-loaded patches were prepared by electrospinning a blend of PCL/ICG, followed by a second layer of the blend made from BSA/polymer. To result in a pliable patch with a high amount of protein, we are aiming at a thickness of 200 to $300 \mu \mathrm{m}$ for the BSA-loaded layer. The required spinning duration, thicknesses and weights of the BSA-layers are shown in table 2. After electrospinning the BSA/PEO-blend $(7.4 \%(\mathrm{w} / \mathrm{w}) / 1.3 \%(\mathrm{w} / \mathrm{w}))$ for 3 hours, a layer with a thickness of $271 \pm 43 \mu \mathrm{m}$ and a weight of $11.8 \pm 1.6 \mathrm{mg}$ was measured. The constant dripping of the blends with PVA and PCL resulted in loss of solution, and therefore a longer electrospinning duration was required, being 5 hours. Still, the weight of the layer was lower compared with the BSA/PEO layer, being in the range of 6.7 to $8.6 \mathrm{mg}$. The BSA/gelatin 
Table 2 Properties and results of the BSA-loaded layer of the electrospun patches used for in vitro tissue soldering using rabbit aorta. Patches with a size of 10 times $20 \mathrm{~mm}$ were cut from the electrospun layer for the measurements. tens=tensile strength; shear

\begin{tabular}{llllllllll}
\hline & & & & & & \\
\end{tabular}

blend was electrospun for 5 hours, due to the lower feed rate of $10 \mu \mathrm{l} / \mathrm{min}$ compared to BSA/PEO with $15 \mu \mathrm{l} / \mathrm{min}$. All layers were flexible and the macroscopic appearance was smooth. The BSA-layers adhered well to the ICG-layer, except for BSA/gelatin, where we could easily pull the BSA-layer off. The removal of the BSA-layer is an unwanted effect, since this complicates the handling of the patch during in vivo surgery. The tensile strength of the layers varies significantly with the PCL/BSA layer (ratio 85/15 (w/w)) tearing at $2895 \pm 357 \mathrm{mN} / \mathrm{mm}^{2}$ and the BSA/PEO (ratio $85 / 15(\mathrm{w} / \mathrm{w})$ ) layer at $97 \pm 25 \mathrm{mN} / \mathrm{mm}^{2}$. Interestingly, layers from BSA/PEO 50/50 (w/w) were much stronger than the $85 / 15(\mathrm{w} / \mathrm{w})$-layers with $709 \pm 122 \mathrm{mN} / \mathrm{mm}^{2}$ which is probably due to its straight fiber morphology instead of beaded fibers that are connected by thin fibers (see figure 2). The tensile strength of the supporting PCL/ICG layer was $3297 \pm 414 \mathrm{mN} / \mathrm{mm}^{2}$, and the tensile strength of the layered patch was specified by this layer and not further affected by the BSA-loaded layer.

\subsection{Shear strength after laser-assisted vascular anastomosis}

For LAVA, the original polymer concentrations in the solvent are irrelevant; instead we report the weight ratios between BSA and each respective auxiliary polymer in the final layer.

Before soldering, the patches made from PVA, PEO and gelatin behaved as follows: The application of the patch was straightforward, they were pliable and could therefore easily be wrapped around the artery. After getting in contact with the humid tissue of the artery, the patches got slightly sticky, enhancing coaption of the two artery stumps. The patch made from BSA/PCL, however, did not become sticky, and the coaption was more difficult.

Figure 1 shows the layered patch made of BSA/PEO before (B) and after LAVA (C). The color of the patch changed from light-green to dark-green and the patch shrank during laser irradiation. The lateral shrinkage was reduced in comparison with the patch soaked in liquid BSA [29]. The surface of the PCL/ICG layer was melting in all cases, a scanning electron micrograph was published previously in [29].

Figure 3 depicts the shear strengths measured after soldering with the different BSA/polymer patches and soldering with a patch soaked in liquid BSA solution. All LAVAs teared at the patch-vessel interface. In LAVAs with BSA-loaded patches made from PVA, PCL, and gelatin low mean shear strengths, ranging from 
Fig 3 Shear strengths received after LAVA for the examined layered patches and ICG-layers soaked in liquid BSA solutions. The native ICG-loaded layer without BSA is depicted as a negative control. The median is represented by the line in the boxes, the fences of the boxes show the lower and upper interquartile range (IQR). The whiskers have the length of $1.5 * I Q R$. The number above the whiskers depicts the average weight of the BSA amount in the patches in $\mathrm{mg}$. We used the following convention for symbols to indicate statistical significance: · : reference, ns: $p \geq 0.05, *: p \leq 0.05, * *: p \leq 0.01, * * *: p \leq 0.001$. To keep the plot clear, the significance was only displayed with the BSA/PEO patch $(85 / 15 \mathrm{w} / \mathrm{w}, 3 \mathrm{~h})$ as a reference group.

2.0 to $4.1 \mathrm{mN} / \mathrm{mm}^{2}$, were measured. LAVAs with the polymer PEO at a ratio of 85/15 (w/w) and a spinning duration of 3 hours exhibit a significantly higher shear strength of $20.7 \pm 4.1 \mathrm{mN} / \mathrm{mm}^{2}$. These values were compared to LAVA with liquid BSA solutions, where we immersed the ICG-loaded layer in BSA dissolved in water at different concentrations. The shear strengths for the 10, 25 and $40 \%$ (w/w) BSA solutions were $0.5 \pm 0.6,11.8 \pm 5.2$ and $20.3 \pm 4.1 \mathrm{mN} / \mathrm{mm}^{2}$, respectively.

Employing PEO allowed us to incorporate $10.1 \pm 1.6 \mathrm{mg}$ of BSA in the patch after a spinning duration of 3 hours. A shorter electrospinning duration of 1 hour reduced the amount of deposited fibers, resulting in a BSA/PEO-layer of $3.8 \pm 0.8 \mathrm{mg}$ with a BSA amount of $3.3 \pm 0.7 \mathrm{mg}$. With this patch we obtained a shear strength of $4.6 \pm 2.3 \mathrm{mN} / \mathrm{mm}^{2}$. A similar shear strength of $3.7 \pm 2.4 \mathrm{mN} / \mathrm{mm}^{2}$ was obtained when the ratio of BSA/PEO was altered to $50 / 50(\mathrm{w} / \mathrm{w})$, where the amount of BSA was $4.9 \pm 1.9 \mathrm{mg}$. Soldering with a soaked patch using a $10 \%(\mathrm{w} / \mathrm{w})$ BSA solution with an amount of $8.3 \pm 3.0 \mathrm{mg}$ BSA, resulted in a shear strength of only $0.5 \pm 0.6 \mathrm{mN} / \mathrm{mm}^{2}$. Patches made from PVA and gelatin yielded low BSA amounts being $4.2 \pm 0.6$ and $3.3 \pm 0.3 \mathrm{mg}$, respectively. We could not bind higher BSA amounts to PVA or gelatin, since they did not dissolve to homogeneous solutions, did not produce fibers and led to clogging of the needle tip.

\subsection{Histological analysis}

Applying a blend of BSA/PEO (85/15 (w/w)) led to a high shear strength of the anastomosis after LAVA. Histological slides from rabbit aorta after LAVA using a layered solder patch, composed of a BSA/PEO layer and a PCL/ICG layer are shown in figure 4. By comparing the native arterial wall and the laser-treated wall, it was evident that thermal damage occurred. The laser-treated Tunica media appears compact and 


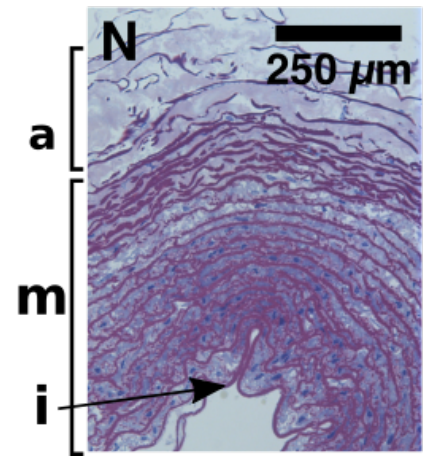

Fig 4 Light micrographs of longitudinal sections of arterial walls immediately after in vitro LAVA using the layered electrospun patch made of BSA/PEO (85/15 (w/w)) and PCL/ICG. A: The image shows the patch closely adhered to the Tunica adventitia and the position were the anastomosis was performed. The layered structure of the patch is visible with the greenish PCL/ICG layer on top and the BSA/PEO layer in contact with the vessel wall. N: The untreated native arterial wall with the Tunica interna (i), and the Tunica media (m), formed by smooth muscle cells and loosely arranged collagen fibers. The Tunica adventitia (a) blends with the connective tissue that surrounds the artery. After LAVA (B and $\mathbf{C}$ ), thermal damage is evident. The Tunica media is densely compressed, while the collagen fibers appear compact and accurately arranged. The Tunica interna with its endothelium seems to be intact.

accurately arranged after soldering, whereas native Tunica media appears loosely arranged. No vacuoles were found in the arterial wall, which is a sign that the temperature did not exceed $100^{\circ} \mathrm{C}$. The thermal damage was restricted to the area that was covered by the patch. Regarding the patch, both structures, the PCL/ICG and the BSA/PEO layer are still visible.

\section{Discussion}

In the first part of this study, the solution and process parameters to achieve stable electrospinning of the BSA/polymer blends were determined. Here "stable" refers to a jet that is uniform, consistent, and not oscillating, the absence of dripping and clogging of the needle tip, and the build-up of a regular, homogeneous scaffold. The electrospinning process is susceptible to many parameters. This includes the composition of the electrospinning solution regarding concentration of the polymer and protein, their molecular weight, viscoelasticity, dielectric properties, and surface tension. Electric potential, gap distance between needle and collector, feed rate and temperature and humidity are referred to as "process parameters" that equally influence the outcome of electrospinning [40]. Although some attempts were made to simulate and hence to accelerate the optimization process, it still remains a "trial-and-error" procedure [52, 53]. Fiber morphologies, namely straight fibers and beaded fibers, have been correlated with different polymer concentrations and low molecular weights, where no chain entanglements were formed [54, 55]. This was confirmed by our results, were we obtained straight or beaded fibers depending on the concentration and ratio of BSA/PEO.

LAVAs performed with high BSA concentrations exhibited a high shear strength compared to those with a low concentration, which is in agreement with the literature $[4,30]$. By involving PCL as an auxiliary 
polymer, it was possible to bind $7.3 \pm 2.2 \mathrm{mg}$ of BSA in a patch. Theoretically this should result in a reasonable strong shear strength. However, the shear strength measured was low, being $2.6 \pm 0.7 \mathrm{mN} / \mathrm{mm}^{2}$. This effect can be explained by the entrapment of BSA inside the hydrophobic PCL fibers, which shields BSA from contacting the tissue. For laser-tissue soldering the direct contact of the BSA with the underlying tissue is however a prerequisite. Long release durations were reported by Valmikinathan et al. [39], who reported a continuous and sustained release of BSA from PCL/BSA fibers over 28 days. Furthermore, an initial burst of BSA from BSA/PCL/Chitosan fibers followed by a gradual release until 14 days was demonstrated [51]. In contrary, PEO, PVA and gelatin are water-soluble polymers where we observed that the BSA-layer starts to dissolve directly after wrapping around the vessel. This guarantees a direct contact of BSA with the tissue. For the maximum shear strength reached with LAVA the mechanical properties of the patches did not play an important role due to the following reasons: First, patches made from PCL, PEO and gelatin melt at the temperature that was used during LAVA. The thickness of the fibers after electrospinning can therefore not explain the different bonding strengths of LAVA. Second, the fracture of the anastomoses during shear strength testing always appeared at the vessel-patch interface, which demonstrates that the strength of the molten patch is not the limiting factor.

LAVA using a layered patch with BSA/PEO $(85 / 15(\mathrm{w} / \mathrm{w}))$ showed similar shear strengths as soldering with a PCL/ICG layer soaked in liquid $40 \%$ (w/w) BSA, with values of $20.7 \pm 4.1 \mathrm{mN} / \mathrm{mm}^{2}$ and $20.3 \pm 4.1 \mathrm{mN} / \mathrm{mm}^{2}$, respectively (see figure 3). This is remarkable, as the amount of BSA is four times lower in the BSA/PEO patch. One explanation for this is the easier positioning of the electrospun BSA/PEO versus the soaked BSA patch. The electrospun patch dissolves and becomes slightly sticky as soon as it gets in contact with the vessel wall. Due to the stickiness, the two vessel stumps hold together, enabling a better contact with the tissue and furthermore enabling a fast and easy preparation of the vessel stumps for LAVA. The same behavior was observed for BSA-loaded patches made from PVA and gelatin. In contrary, the patch soaked in liquid BSA is sticky from the beginning, and therefore more difficult to handle. The maximum load before tearing apart obtained with sutured rabbit aortas was higher compared to our novel layered patch, with $2042 \pm 112 \mathrm{mN}$ and $1321 \pm 265 \mathrm{mN}$, respectively. However, the same study demonstrated that the lower bonding strength still withstands blood flow [29].

The extent of the thermal damage was found to be similar for all BSA/polymer patches used, since all LAVA experiments were performed by heating the patches for $30 \mathrm{~s}$ at a temperature range between $75^{\circ} \mathrm{C}$ and $85^{\circ} \mathrm{C}$. The heating process was controlled by a thermal camera, and described in detail in Schönfeld et al. [29]. By comparing the histologies of the layered BSA/PEO patch (figure 4) with the patch soaked in liquid albumin [29], a comparable extent of thermal damage was visible. The Tunica interna with its endothelium, which plays a crucial role for the function of blood flow [56], appeared structurally intact. The layered structures of the patch, the greenish PCL/ICG layer and the BSA/PEO layer, are still visible after LAVA, showing that they do not interfuse completely.

Pre-clinical trials, where we tested the handling of the layered BSA/PEO patch in a realistic setup, demonstrated the advantages of this patch compared to a patch that was soaked in liquid BSA. Due to the dry state of the patch, storage and transport is uncomplicated and does not require continuous cooling. During surgery, the patch can be easily cut to the required size. The patch is pliable, mechanically stable, and can be handled easily. It is of special importance, that the layered patch becomes slightly sticky during its application to the vessels, allowing for a precise wrapping of the patch around the vessels and for a precise and fast approximation of the vessel stumps. Therefore, by using this patch, we assume a decreased failure rate of LAVA. An additional advantage may be the improvement of the biophysical and chemical stability of BSA by PEO, an effect that is also used for drug delivery [57]. The performance of the BSA/PEO patch during surgery, the long-term effects during healing-phase and the shelf-life of the patch have to be further tested. 


\section{Conclusions}

Electrospinning is a relatively simple technique and enables the entrapment of proteins in polymer fibers. In this study, BSA was successfully encapsulated in microfibers with PEO, PCL, PVA and gelatin using a blend electrospinning technique. The BSA/PEO blend was outstanding regarding the stability of the electrospinning process. Using such a BSA/PEO (ratio 85/15 (w/w)) blend, high BSA-loading was possible, leading to shear strengths after LAVA, which are comparable to LAVA performed with a patch soaked in liquid albumin solution. The BSA/PEO patches however enabled a much easier handling resulting in a fast and tight coadaptation and preparation for LAVA during surgery. In comparison, patches from PVA, PCL, and gelatin were limited in BSA content, which in turn led to a lower bonding strength after LAVA. Our findings support the potential of electrospun scaffolds with protein loading in biomedical applications.

\section{Acknowledgments}

This research was funded by the Swiss National Science Foundation (project number 32003B-133083). Dieter Bosshardt and Silvia Owusu from the Robert K. Schenk Laboratory of Oral Histology, University of Bern are acknowledged for histological analysis. The authors thank René Nyffenegger for his technical assistance, Michael Stoffel, and Pascal Moll for their support. We thank Kani-Swiss GmbH, Geltwil, Switzerland, for providing us the rabbit aortas.

\section{References}

[1] Lawrence S. Bass and Michael R. Treat. Laser tissue welding: A comprehensive review of current and future. Lasers in Surgery and Medicine, 17(4):315-349, 1995. ISSN 01968092. doi: 10.1002/1sm. 1900170402. URL http://doi.wiley.com/10.1002/1sm.1900170402.

[2] I.C.D.Y.M. Wolf -de Jonge, J.F. Beek, and R. Balm. 25 Years of Laser Assisted Vascular Anastomosis (LAVA): What Have We Learned? European Journal of Vascular and Endovascular Surgery, 27(5):466-476, may 2004. ISSN 10785884. doi: 10.1016/j.ejvs.2004.02.021. URL http://linkinghub.elsevier.com/retrieve/pii/s1078588404001157.

[3] Beat Ott, Mihai A. Constantinescu, Dominique Erni, Andrej Banic, Thomas Schaffner, and Martin Frenz. Intraluminal laser light source and external solder: In vivo evaluation of a new technique for microvascular anastomosis. Lasers in Surgery and Medicine, 35(4):312-316, oct 2004. ISSN 01968092. doi: 10.1002/1sm.20096. URL http: / / doi.wiley.com/10.1002/1sm.20096.

[4] Karen M McNally, Brian S Sorg, Ashley J Welch, Judith M Dawes, and Earl R Owen. Photothermal effects of laser tissue soldering. Physics in Medicine and Biology, 44(4):983-1002, apr 1999. ISSN 0031-9155. doi: 10.1088/0031-9155/44/4/013. URL http://stacks.iop.org/0031-9155/ $44 / i=4 / a=013$ ? key=crossref . cd4e823063a 64 ef34f28373f56339483.

[5] Brian D. Byrd, Douglas L./Heintzelman, and Karen M. McNally-Heintzelman. Absorption properties of alternative chromophores for use in laser tissue soldering applications. Biomedical sciences instrumentation, 39:6-11, 2003. ISSN 0067-8856. URL http://www.ncbi.nlm.nih.gov/ pubmed/12724860.

[6] Giuseppe Esposito, Francesca Rossi, Paolo Matteini, Alba Scerrati, Alfredo Puca, Alessio Albanese, Giacomo Rossi, Fulvio Ratto, Giulio Maira, and Roberto Pini. In vivo laser assisted microvascular repair and end-to-end anastomosis by means of indocyanine green-infused chitosan patches: A pilot study. Lasers in Surgery and Medicine, 45(5):318-325, jul 2013. ISSN 01968092. doi: 10.1002/lsm. 22145. URL http://doi.wiley.com/10.1002/1.sm.22145. 
[7] Daniel S. Schöni, Serge Bogni, Amadé Bregy, Amina Wirth, Andreas Raabe, Istvan Vajtai, Uwe Pieles, Michael Reinert, and Martin Frenz. Nanoshell assisted laser soldering of vascular tissue. Lasers in Surgery and Medicine, 43(10):975-983, dec 2011. ISSN 01968092. doi: 10.1002/1sm.21140. URL http://doi.wiley.com/10.1002/lsm.21140.

[8] Dara R. Pabittei, Michal Heger, Ron Balm, Han E. H. Meijer, Bas de Mol, and Johan F. Beek. Electrospun Poly(-Caprolactone) Scaffold for Suture-Free Solder-Mediated Laser-Assisted Vessel Repair. Photomedicine and Laser Surgery, 29(1):19-25, jan 2011. ISSN 1549-5418. doi: 10.1089/pho.2010. 2779. URL http://www. liebertonline.com/doi/abs/10.1089/pho.2010.2779.

[9] Brian S. Sorg, Karen M. McNally, and Ashley J. Welch. Biodegradable polymer film reinforcement of an indocyanine green-doped liquid albumin solder for laser-assisted incision closure. Lasers in Surgery and Medicine, 27(1):73-81, jan 2000. ISSN 0196-8092. doi: 10.1002/1096-9101(2000) 27:1〈73::AID-LSM10〉3.0.CO;2-7. URL http://doi.wiley.com/10.1002/ $1096-9101\left\{\frac{\circ}{2} 282000\left\{\frac{\circ}{\circ}\right\} 2927\{\%\} 3 A 1\{\%\} 3 C 73\{\%\} 3 A\{\%\} 3 A A I D-L S M 10\{\%\} 3 E 3\right.$. $0 . \mathrm{CO}\{\circ\} 3 \mathrm{~B} 2-7$.

[10] Wei Wang. Instability, stabilization, and formulation of liquid protein pharmaceuticals. International Journal of Pharmaceutics, 185(2):129-188, aug 1999. ISSN 03785173. doi: 10.1016/ S0378-5173(99)00152-0. URL http://linkinghub.elsevier.com/retrieve/pii/ S0378517399001520.

[11] Andreas Greiner and Joachim H. Wendorff. Electrospinning: A Fascinating Method for the Preparation of Ultrathin Fibers. Angewandte Chemie International Edition, 46(30):5670-5703, jul 2007. ISSN 14337851. doi: 10.1002/anie.200604646. URL http://doi.wiley.com/10.1002/anie. 200604646 .

[12] Subramanian Sundarrajan, Kwong Luck Tan, Soon Huat Lim, and Seeram Ramakrishna. Electrospun Nanofibers for Air Filtration Applications. Procedia Engineering, 75:159-163, 2014. ISSN 18777058. doi: 10.1016/j.proeng.2013.11.034. URL http: / / inkinghub.elsevier.com/retrieve/ pii/S1877705813018092.

[13] Seema Agarwal, Andreas Greiner, and Joachim H. Wendorff. Functional materials by electrospinning of polymers. Progress in Polymer Science, 38(6):963-991, jun 2013. ISSN 00796700. doi: 10.1016/ j.progpolymsci.2013.02.001. URL http: / / inkinghub.elsevier.com/retrieve/pii/ S0079670013000087.

[14] N. M. Bedford and A. J. Steckl. Photocatalytic Self Cleaning Textile Fibers by Coaxial Electrospinning. ACS Applied Materials \& Interfaces, 2(8):2448-2455, aug 2010. ISSN 1944-8244. doi: 10.1021/am1005089. URL http://pubs.acs.org/doi/abs/10 .1021/am1005089.

[15] Quynh P Pham, Upma Sharma, and Antonios G Mikos. Electrospinning of Polymeric Nanofibers for Tissue Engineering Applications: A Review. Tissue Engineering, 12(5):1197-1211, may 2006. ISSN 1076-3279. doi: 10.1089/ten.2006.12.1197. URL http://www.liebertonline.com/doi/ abs/10.1089/ten.2006.12.1197.

[16] Nae Gyune Rim, Choongsoo S Shin, and Heungsoo Shin. Current approaches to electrospun nanofibers for tissue engineering. Biomedical Materials, 8(1):014102, feb 2013. ISSN 1748-6041. doi: 10.1088/ 1748-6041/8/1/014102. URL http://stacks.iop.org/1748-605x/8/i=1/a=014102. 
[21] Martina Abrigo, Sally L. McArthur, and Peter Kingshott. Electrospun Nanofibers as Dressings for Chronic Wound Care: Advances, Challenges, and Future Prospects. Macromolecular Bioscience, 14(6):772-792, jun 2014. ISSN 16165187. doi: 10.1002/mabi.201300561. URL http://doi . wiley.com/10.1002/mabi.201300561.

[22] Anne J. Meinel, Oliver Germershaus, Tessa Luhmann, Hans P. Merkle, and Lorenz Meinel. Electrospun matrices for localized drug delivery: Current technologies and selected biomedical applications. European Journal of Pharmaceutics and Biopharmaceutics, 81(1):1-13, may 2012. ISSN 09396411. doi: 10.1016/j.ejpb.2012.01.016. URL http://linkinghub.elsevier.com/retrieve/ pii/s0939641112000379.

[23] Gareth R Williams, Nicholas P Chatterton, Tahir Nazir, Deng-Guang Yu, Li-Min Zhu, and Christopher J Branford-White. Electrospun nanofibers in drug delivery: recent developments and perspectives. Therapeutic Delivery, 3(4):515-533, apr 2012. ISSN 2041-5990. doi: 10.4155/tde.12.17. URL http://www. future-science.com/doi/10.4155/tde.12.17.

[24] Seeram Ramakrishna, Maedeh Zamani, and Molamma P Prabhakaran. Advances in drug delivery via electrospun and electrosprayed nanomaterials. International Journal of Nanomedicine, 8(1):2997, aug 2013. ISSN 1178-2013. doi: 10.2147/IJN.S43575. URL http://www. dovepress.com/ advances-in-drug-delivery-via-electrospun-and-electrosprayed-nanomater-peer-re

[25] Young Ju Son, Woo Jin Kim, and Hyuk Sang Yoo. Therapeutic applications of electrospun nanofibers for drug delivery systems. Archives of Pharmacal Research, 37(1):69-78, jan 2014. ISSN 02536269. doi: 10.1007/s12272-013-0284-2. URL http://link.springer.com/10.1007/ s12272-013-0284-2.

[26] Travis J. Sill and Horst A. von Recum. Electrospinning: Applications in drug delivery and tissue engineering. Biomaterials, 29(13):1989-2006, may 2008. ISSN 01429612. doi: 10.1016/ j.biomaterials.2008.01.011. URL http://linkinghub.elsevier.com/retrieve/pii/ S0142961208000203. 
[27] Suwan N Jayasinghe. Cell electrospinning: a novel tool for functionalising fibres, scaffolds and membranes with living cells and other advanced materials for regenerative biology and medicine. The Analyst, 138(8):2215-2223, 2013. ISSN 0003-2654. doi: 10.1039/c3an36599a. URL http: //xlink.rsc.org/?DOI=c3an36599a.

[28] Samantha L. Sampson, Luisa Saraiva, Kenth Gustafsson, Suwan N. Jayasinghe, and Brian D. Robertson. Cell Electrospinning: An In Vitro and In Vivo Study. Small, 10(1):78-82, jan 2014. ISSN 16136810. doi: 10.1002/smll.201300804. URL http://doi.wiley.com/10.1002/smll. 201300804 .

[29] Annemarie Schönfeld, Zacharia Mbädjol Kabra, Mihai Constantinescu, Dieter Bosshardt, Michael H. Stoffel, Kirsten Peters, and Martin Frenz. Binding of indocyanine green in polycaprolactone fibers using blend electrospinning for in vivo laser-assisted vascular anastomosis. Lasers in Surgery and Medicine, 49(10):928-939, jul 2017. ISSN 01968092. doi: 10.1002/1sm.22701. URL http:// doi.wiley.com/10.1002/1sm.22701.

[30] Amadé Bregy, Serge Bogni, Vianney J.P. Bernau, Istvan Vajtai, Felix Vollbach, Alke Petri-Fink, Mihai Constantinescu, Heinrich Hofmann, Martin Frenz, and Michael Reinert. Solder doped polycaprolactone scaffold enables reproducible laser tissue soldering. Lasers in Surgery and Medicine, 40(10): 716-725, dec 2008. ISSN 01968092. doi: 10.1002/lsm.20710. URL http: / / doi • wiley • com/ $10.1002 / 1 \mathrm{sm} .20710$.

[31] Tomasz Kowalczyk, Aleksandra Nowicka, Danek Elbaum, and Tomasz A. Kowalewski. Electrospinning of Bovine Serum Albumin. Optimization and the Use for Production of Biosensors. Biomacromolecules, 9(7):2087-2090, jul 2008. ISSN 1525-7797. doi: 10.1021/bm800421s. URL http: //pubs.acs.org/doi/abs/10.1021/bm800421s.

[32] Rouhollah Mehdinavaz Aghdam, Siamak Najarian, Saeed Shakhesi, Samaneh Khanlari, Keyvan Shaabani, and Shahriar Sharifi. Investigating the effect of PGA on physical and mechanical properties of electrospun PCL/PGA blend nanofibers. Journal of Applied Polymer Science, 124(1):123-131, apr 2012. ISSN 00218995. doi: 10.1002/app.35071. URL http://doi.wiley.com/10.1002/ app. 35071.

[33] Christina Tang, A. Evren Ozcam, Brendon Stout, and Saad A. Khan. Effect of pH on Protein Distribution in Electrospun PVA/BSA Composite Nanofibers. Biomacromolecules, 13(5):1269-1278, may 2012. ISSN 1525-7797. doi: 10.1021/bm2017146. URL http://pubs.acs.org/doi/abs/ $10.1021 / \mathrm{bm} 2017146$.

[34] Marina I. Santos, Ronald E. Unger, Rui A. Sousa, Rui L. Reis, and C. James Kirkpatrick. Crosstalk between osteoblasts and endothelial cells co-cultured on a polycaprolactone-starch scaffold and the in vitro development of vascularization. Biomaterials, 30(26):4407-4415, sep 2009. ISSN 01429612. doi: 10.1016/j.biomaterials.2009.05.004. URL http://linkinghub. elsevier. com/retrieve/pii/s0142961209004797.

[35] Hong Zhang, ChenGuang Zhao, Yunhui Zhao, Gongwen Tang, and Xiaoyan Yuan. Electrospinning of ultrafine core/shell fibers for biomedical applications. Science China Chemistry, 53(6):1246-1254, jun 2010. ISSN 1674-7291. doi: 10.1007/s11426-010-3180-3. URL http: / / ink . springer. $\mathrm{com} / 10.1007 / \mathrm{s} 11426-010-3180-3$.

[36] Honglin Qu, Suying Wei, and Zhanhu Guo. Coaxial electrospun nanostructures and their applications. Journal of Materials Chemistry A, 1(38):11513, 2013. ISSN 2050-7488. doi: 10.1039/c3ta12390a. URL http: / / xlink.rsc.org/ ?DOI=c3ta12390a. 
[37] Xiaoqiang Li, Yan Su, Shuiping Liu, Lianjiang Tan, Xiumei Mo, and Seeram Ramakrishna. Encapsulation of proteins in poly(l-lactide-co-caprolactone) fibers by emulsion electrospinning. Colloids and Surfaces B: Biointerfaces, 75(2):418-424, feb 2010. ISSN 09277765. doi: 10. 1016/j.colsurfb.2009.09.014. URL http: / / inkinghub.elsevier.com/retrieve/pii/ S0927776509004469.

[38] Ye Yang, Xiaohong Li, Wenguo Cui, Shaobing Zhou, Rui Tan, and Chaoyang Wang. Structural stability and release profiles of proteins from coreshell poly (DLlactide) ultrafine fibers prepared by emulsion electrospinning. Journal of Biomedical Materials Research Part A, 86A(2):374-385, aug 2008. ISSN 15493296. doi: 10.1002/jbm.a.31595. URL http://doi.wiley.com/10.1002/jbm . a. 31595 .

[39] Chandra M. Valmikinathan, Steven Defroda, and Xiaojun Yu. Polycaprolactone and Bovine Serum Albumin Based Nanofibers for Controlled Release of Nerve Growth Factor Biomacromolecules, 10 (5):1084-1089, may 2009. ISSN 1525-7797. doi: 10.1021/bm8012499. URL http: / / pubs . acs . org/doi/abs/10.1021/bm8012499.

[40] Ying Yang, Zhidong Jia, Qiang Li, and Zhicheng Guan. Experimental investigation of the governing parameters in the electrospinning of polyethylene oxide solution. IEEE Transactions on Dielectrics and Electrical Insulation, 13(3):580-585, jun 2006. ISSN 1070-9878. doi: 10.1109/TDEI.2006. 1657971. URL http: / / ieeexplore. ieee.org/document/1657971/.

[41] J Deitzel. Controlled deposition of electrospun poly(ethylene oxide) fibers. Polymer, 42(19): 8163-8170, sep 2001. ISSN 00323861. doi: 10.1016/S0032-3861(01)00336-6. URL http: //linkinghub.elsevier.com/retrieve/pii/s0032386101003366.

[42] Y Mohammadi, M Soleimani, M Fallahi-Sichani, a Gazme, V Haddadi-Asl, E Arefian, J Kiani, R Moradi, a Atashi, and N Ahmadbeigi. Nanofibrous poly(epsilon-caprolactone)/poly(vinyl alcohol)/chitosan hybrid scaffolds for bone tissue engineering using mesenchymal stem cells. The International journal of artificial organs, 30(3):204-11, mar 2007. ISSN 0391-3988. URL http: //www.ncbi.nlm.nih.gov/pubmed/17417759.

[43] Jun Zeng, Achim Aigner, Frank Czubayko, Thomas Kissel, Joachim H. Wendorff, and Andreas Greiner. Poly(vinyl alcohol) Nanofibers by Electrospinning as a Protein Delivery System and the Retardation of Enzyme Release by Additional Polymer Coatings. Biomacromolecules, 6(3):1484-1488, may 2005. ISSN 1525-7797. doi: 10.1021/bm0492576. URL http://pubs.acs.org/doi/ $\mathrm{abs} / 10.1021 / \mathrm{bm} 0492576$.

[44] Y. Wang and Y.-L. Hsieh. Immobilization of lipase enzyme in polyvinyl alcohol (PVA) nanofibrous membranes. Journal of Membrane Science, 309(1-2):73-81, feb 2008. ISSN 03767388. doi: 10. 1016/j.memsci.2007.10.008. URL http://linkinghub.elsevier.com/retrieve/pii/ S 0376738807007326.

[45] Achim Salamon, Sandra van Vlierberghe, Ine van Nieuwenhove, Frank Baudisch, Geert-Jan Graulus, Verena Benecke, Kristin Alberti, Hans-Georg Neumann, Joachim Rychly, José Martins, Peter Dubruel, and Kirsten Peters. Gelatin-Based Hydrogels Promote Chondrogenic Differentiation of Human Adipose Tissue-Derived Mesenchymal Stem Cells In Vitro. Materials, 7(2):1342-1359, feb 2014. ISSN 1996-1944. doi: 10.3390/ma7021342. URL http://www.mdpi.com/1996-1944/7/ 2/1342/. 
[46] Zuwei Ma, Wei He, Thomas Yong, and S Ramakrishna. Grafting of Gelatin on Electrospun Poly(caprolactone) Nanofibers to Improve Endothelial Cell Spreading and Proliferation and to Control Cell Orientation. Tissue Engineering, 11(7-8):1149-1158, jul 2005. ISSN 1076-3279. doi: 10.1089/ten.2005.11.1149. URL http://www.liebertonline.com/doi/abs/10.1089/ ten.2005.11.1149.

[47] Yanzhong Zhang, Hongwei Ouyang, Chwee Teck Lim, Seeram Ramakrishna, and Zheng-Ming Hụang. Electrospinning of gelatin fibers and gelatin/PCL composite fibrous scaffolds. Journal of Biomedical Materials Research, 72B(1):156-165, jan 2005. ISSN 0021-9304. doi: 10.1002/jbm.b.30128. URL http://doi.wiley.com/10.1002/jbm.b.30128.

[48] Wei Fu, Zhenling Liu, Bei Feng, Renjie Hu, Xiaomin He, Hao Wang, Meng Yin, Huimin Huang, Haibo Zhang, and Wei Wang. Electrospun gelatin/PCL and collagen/PLCL scaffolds for vascular tissue engineering. International Journal of Nanomedicine, 9(1):2335, may 2014. ISSN 1178-2013. doi: 10.2147/IJN.S61375. URL http://www.dovepress.com/ electrospun-gelatinpcl-and-collagenplcl-scaffolds-for-vascular-tissue--peer-re

[49] Johannes Schindelin, Ignacio Arganda-Carreras, Erwin Frise, Verena Kaynig, Mark Longair, Tobias Pietzsch, Stephan Preibisch, Curtis Rueden, Stephan Saalfeld, Benjamin Schmid, Jean-Yves Tinevez, Daniel James White, Volker Hartenstein, Kevin Eliceiri, Pavel Tomancak, and Albert Cardona. Fiji: an open-source platform for biological-image analysis. Nature Methods, 9(7):676-682, jun 2012. ISSN 1548-7091. doi: 10.1038/nmeth.2019. URL http://www. nature.com/doifinder/ $10.1038 /$ nmeth. 2019 .

[50] R Core Team, R core Team, and R Development Core Team. R: A language and environment for statistical computing. $R$ : A language and environment for statistical computing., 2015. ISSN 3-900051$07-0$.

[51] Fatemeh Roozbahani, Naznin Sultana, Davood Almasi, and Farnaz Naghizadeh. Effects of Chitosan Concentration on the Protein Release Behaviour of Electrospun Poly $(\epsilon$-caprolactone $) /$ Chitosan Nanofibers. Journal of Nanomaterials, 2015:1-11, apr 2015. ISSN 1687-4110. doi: 10.1155/2015/ 747420. URL http://www. hindawi.com/journals/jnm/2015/747420/.

[52] H. Mohammad Khanlou, B. Chin Ang, S. Talebian, A. Muhammad Afifi, and A. Andriyana. Electrospinning of polymethyl methacrylate nanofibers: optimization of processing parameters using the Taguchi design of experiments. Textile Research Journal, 85(4):356-368, mar 2015. ISSN 00405175. doi: 10.1177/0040517514547208. URL http://trj.sagepub.com/cgi/doi/10 . $1177 / 0040517514547208$.

[53] Stuart R. Coles, Daniel K. Jacobs, James O. Meredith, Guy Barker, Andrew J. Clark, Kerry Kirwan, Jon Stanger, and Nick Tucker. A design of experiments $(\mathrm{DoE})$ approach to material properties optimization of electrospun nanofibers. Journal of Applied Polymer Science, 117(4):2251-2257, aug 2010. ISSN 00218995. doi: 10.1002/app.32022. URL http://doi .wiley. com/10.1002/app. 32022.

[54] Joachim H. Wendorff, Seema Agarwal, and Andreas. Greiner. Electrospinning. Wiley-VCH Verlag GmbH \& Co. KGaA, Weinheim, Germany, 1. edition, apr 2012. ISBN 9783527647705. doi: 10.1002/ 9783527647705. URL http://doi.wiley.com/10.1002/9783527647705.

[55] Matthew G. McKee, Garth L. Wilkes, Ralph H. Colby, and Timothy E. Long. Correlations of Solution Rheology with Electrospun Fiber Formation of Linear and Branched Polyesters. Macromolecules, 37 (5):1760-1767, mar 2004. ISSN 0024-9297. doi: 10.1021/ma035689h. URL http: / pubs . acs . org/doi/abs/10.1021/ma035689h. 
[56] Peramaiyan Rajendran, Thamaraiselvan Rengarajan, Jayakumar Thangavel, Yutaka Nishigaki, Dhanapal Sakthisekaran, Gautam Sethi, and Ikuo Nishigaki. The Vascular Endothelium and Human Diseases. International Journal of Biological Sciences, 9(10):1057-1069, 2013. ISSN 1449-2288. doi: 10.7150/ijbs.7502. URL http: / /www. i jbs . com/v09p1057.htm.

[57] S. Dhawan, K Dhawan, M Varma, and V R Sinha. Applications of Poly (ethylene oxide) in drug delivery systems. Pharmaceutical Technology, 29(9):82-96, 2005. URL www • pharmtech. com. 